\title{
ACTIVIDAD FÍSICA Y PERCEPCIÓN DE ESFUERZO POR GÉNERO EN EDUCACIÓN FÍSICA Y RECREO DE ESCOLARES MEXICANOS
}

\section{PHYSICAL ACTIVITY AND PERCEPTION OF EFFORT BY GENDER IN PHYSICAL EDUCATION AND RECESS OF MEXICAN SCHOOLCHILDREN}

\section{ATIVIDADE FÍSICA E PERCEPÇÃO DE ESFORÇO POR GÊNERO EM EDUCAÇÃO FÍSICA E RECREIO DE ESCOLARES MEXICANOS}

\author{
Javier Arturo Hall-López ${ }^{1}$ \\ Paulina Yesica Ochoa-Martínez ${ }^{2}$ \\ Fernando Meza Correa ${ }^{3}$ \\ Cristhian Emmanuel López Campos ${ }^{4}$ \\ Pedro Sáenz-López Buñuel ${ }^{5}$
}

1 Profesor Investigador de la Facultad de Deportes Campus Mexicali, Universidad Autónoma de Baja California. México. Cuerpo Académico Ciencias de la Actividad Física y Deporte UABC 175. Licenciatura en Educación Física, Escuela Superior de Educación Física, Universidad Autónoma de Sinaloa, Maestría en Ciencias del Deporte opción Biología, Universidad Autónoma de Chihuahua. Doctorado en Medicina del Deporte Universidad Católica "Nuestra Señora de la Asunción". E-mail: javierhall@uabc.edu.mx 
2 Profesora Investigador de la Facultad de Deportes Campus Mexicali, Universidad Autónoma de Baja California. México. Cuerpo Académico Ciencias de la Actividad Física y Deporte UABC 175. Licenciatura en Educación Física y Maestría en Ciencias del Deporte opción Biología, Universidad Autónoma de Chihuahua. Doctorado en Medicina del Deporte Universidad Católica "Nuestra Señora de la Asunción". E-mail: pochoa@uabc.edu.mx

3 Profesor de la Secretaria de Educación Publica México. Licenciado en Actividad Física y Deporte egresado de la Facultad de Deportes Universidad Autónoma de Baja California. Licenciado en Actividad Física y Deporte egresado de la Facultad de Deportes Universidad Autónoma de Baja California. E-mail: mezaf53@uabc.edu.mx

4 Profesor de la Facultad de Deportes Universidad Autónoma de Baja California México. Licenciado en Actividad Física y Deporte egresado de la Facultad de Deportes Universidad Autónoma de Baja California. Maestría en Educación Física y Deporte Escolar egresado de la Facultad de Deportes Universidad Autónoma de Baja California. E-mail: lopez. cristhian@uabc.edu.mx

5 Catedrático del Departamento de Didácticas Integradas, Universidad de Huelva, España. Licenciado en Actividad Física y Deporte Universidad de Granada. Doctorado en Educacion Universidad de Sevilla. E-mail: psaenz@uhu.es

Hall-López, J. H.; Ochoa-Martínez, P. Y; Meza Correa, F.; López Campos, C. E. \& Sáenz-López Buñuel, P. (2017). Actividad Física Y Percepción De Esfuerzo Por Género En Educación Física Y Recreo De Escolares Mexicanos. Educación Física y Deporte, 36(2), 219-243. Jul.-Dic. http://doi.org/10.17533/udea.efyd.v36n2a04 


\section{RESUMEN}

Problema: evidencia científica relaciona, por género, mayor sedentarismo en niñas. Objetivo: comparar, por género, la actividad física moderada a vigorosa, la tasa de gasto energético, la frecuencia cardiaca y la percepción de esfuerzo en estudiantes de tercero y cuarto grado de primaria durante la clase de educación física y el recreo. Método: participaron

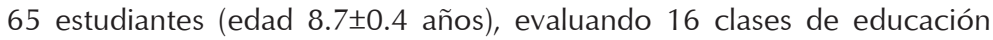
física y recreo, utilizando como instrumentos el sistema para observar el tiempo de instrucción de actividad física, el pulsómetro polar ${ }^{\circledR}$ y la tabla de clasificación gráfica de esfuerzo para niños. Resultados: el test t-Student reportó diferencias significativas en educación física con mayores promedios en niños que en niñas, de actividad física moderada a vigorosa (0.001), tasa de gasto energético (0.002) y frecuencia cardiaca (0.005). Conclusión: con base en los resultados, se concluye que la intensidad de la actividad física es mayor en los niños; es importante retroalimentar la manera de impartir la clase de educación física, con estrategias didácticas y contenidos que, equitativamente, involucren a las niñas en actividad física.

PALABRAS CLAVE: Educación y Entrenamiento Físico, Educación, Identidad de Género, Recreación.

\section{ABSTRACT}

Problem: Scientific evidence related to gender indicates a greater sedentary lifestyle in girls. Objective: To compare moderate and high physical activity by gender, energy expenditure rate, heart rate and the effort perception among students in third and fourth grade in elementary school during physical education class and recess. Method: 65 students participated (age $8.7 \pm 0.4$ ) evaluating 16 physical education classes and recess using as instruments the system to observe the physical activity instruction time, polar heart rate monitor ${ }^{\circledR}$ and the graphical effort classification table for children. Results: The t-Student test reported significant differences in physical education with higher averages in boys than girls with moderate to high intensity physical activity (0.001), energy expenditure rate (0.002) and heart rate (0.005). Conclusion: Considering the results, the study concludes that the intensity of physical activity is greater in boys, therefore it is important to provide feedback on how to improve physical education classes, with didactic strategies and contents that involve girls equally in physical activity.

KEYWORDS: Physical Education and Training, Education, Gender Identity, Leisure. 


\section{RESUMO:}

Problema: Evidência científica relaciona por gênero maior sedentarismo em meninas. Objetivo: Comparar por gênero a atividade física moderada a vigorosa, taxa de despesa energética, frequência cardíaca e percepção de esforço em estudantes de terceiro e quarto grau de primária durante a classe de educação física e o recreio. Método: Participaram 65 estudantes (idade $8.7 \pm 0.4$ anos) avaliando 16 classes de educação física e recreio utilizando como instrumentos o sistema para observar o tempo de instrução de atividade física, pulsómetro polar ${ }^{\circledR}$ e a tabela de classificação gráfica de esforço para meninos. Resultados: $\mathrm{O}$ teste t-Student reporto diferenças significativas em educação física com maiores médias em meninos que meninas de atividade física moderada a vigorosa (0.001), taxa de despesa energética (0.002) e frequência cardíaca (0.005). Conclusão: Com base nos resultados conclui-se que a intensidade da atividade física é maior nos meninos, é importante retroalimentar a maneira de dar a classe de educação física com estratégias didáticas e conteúdos que eqüitativamente envolvam as meninas em atividade físicas.

PALAVRAS-CHAVE: Educação e Treinamento Físico, Educação, Identidade de Gênero, Recreação. 


\section{INTRODUCCIÓN}

El término sedentarismo proviene del latín sedentarios, que significa estar sin moverse o permanecer en el mismo lugar (Yates et al., 2011). Revisiones sistemáticas y metaanálisis, sugieren que niños y niñas sedentarios presentan en mayor medida riesgo a padecer patologías asociadas a la obesidad (Brooke et al., 2014; Sims et al., 2015). La Organización Mundial de la Salud (OMS) recomienda que las personas entre 5 a 17 años de edad, dediquen como mínimo 60 minutos diarios en actividad física de intensidad moderada a vigorosa (WHO, 2014), las mismas que se recomiendan en México por medio de las guías alimentarias y de actividad física en contexto de sobrepeso y obesidad en la población mexicana (Bonvecchio et al., 2015). En nuestro país, la Encuesta Nacional de Salud y Nutrición de Medio Camino ENSANUT MC 2016 (INSP, 2016) refiere que el 17.2\% de los niños $(21.8 \%)$ y niñas $(12.7 \%)$ de entre $10-14$ años de edad, se categorizan como activos de acuerdo a los criterios de la OMS. También la ENSANUT MC 2016, reportó del año 2012 a 2016 una disminución del $34.4 \%$ a $33.2 \%$ en la prevalencia combinada de sobrepeso y obesidad en niños de edad escolar; sin embargo, dicha prevalencia no se redujo en mujeres, sino únicamente en hombres con sobrepeso (INSP, 2016).

A nivel mundial, se ha identificado como área de oportunidad a la educación básica para promover un estilo de vida activo y saludable desde edades tempranas (Kim, 2012), pues, por su amplia cobertura, es un medio ideal para la promoción y desarrollo de actitudes positivas y hábitos saludables, que sirvan de base para que los niños y adolescentes tiendan a adoptar un estilo de vida activo como adultos (Langford et al., 2015; Lonsdale et al., 2013) y, de esta manera, mejorar la calidad de vida y reducir los costos en el tratamiento de enfermedades crónicas no trasmisibles (Kim, 2012). Dado que, de los componentes del gasto energético, la actividad física es el único que puede ser 
modificado de manera voluntaria (Honas et al., 2008), en el ámbito escolar, las clases de educación física son el espacio ideal para fomentar la práctica de actividad física (Story et al., 2009), debido a que, en la educación física, el enfoque pedagógico implica movimiento con esfuerzo e intensidades que, en ocasiones, corresponden a un nivel de actividad física moderada a vigorosa, como aquella que requiere un gasto energético de entre 3 a 6 MET (equivalentes metabólicos) (WHO, 2014).

La intensidad de la actividad física se define como la rapidez o magnitud del esfuerzo requerido para realizar un gasto energético por medio del sistema músculo esquelético. Se determina en relación con el gasto energético y el esfuerzo físico contra alguna resistencia, dificultando el trabajo sobre el cuerpo, en un periodo de tiempo determinado (Honas et al., 2008). En educación física, los profesores pueden establecer acciones pedagógicas y de esfuerzo físico que adhieran al estudiante en actividades divertidas, placenteras, que induzcan la práctica de actividad física extraescolar (Shilton, 2008; Lonsdale et al., 2013). Aunque la intensidad, el gasto energético y el esfuerzo físico de la actividad física no son los únicos factores para valorar la enseñanza de la educación física, de acuerdo con estándares establecidos internacionalmente por la National Association for Sport and Physical Education (NASPE), se recomienda que, en las sesiones de educación física, el estudiante participe en actividades físicas con al menos una intensidad moderada a vigorosa, por arriba del 50\% del tiempo de la sesión, es decir, con un gasto energético similar a caminar o correr (Banville, 2006; NASPE, 2009).

Con base en un modelo socio ecológico, existen múltiples factores ambientales que pueden determinar la intensidad de la actividad física de los estudiantes durante la jornada escolar. Investigaciones de tipo transversal y longitudinal, mencionan que, dentro de estos factores, está la capacitación del profesor, el tamaño del grupo, la edad de los estudiantes, el horario, el mate- 
rial, los instrumentos e instalaciones con las que cuente el centro escolar, y la duración de las clases (Brooke et al., 2014; Hollis et al., 2016; Skala et al., 2012), siendo importantes el modelo educativo, el contexto escolar de cada país (McKenzie \& van der Mars, 2015) y el estilo de vida activo que el estudiante lleve en su entorno familiar (McKenzie et al., 2008). Estudios que han evaluado la percepción de esfuerzo en la clase de educación física, concluyeron que las niñas reportaron mayor esfuerzo que los niños (Hernández et al., 2010). Otras investigaciones indican, en diferentes niveles educativos, que durante las clases de educación física, los niños varones son físicamente más activos y realizan tareas que implican mayor movimiento que las niñas (Nettlefold et al., 2011; Smith et al., 2014).

En el ámbito escolar, existen diferentes instrumentos para medir la intensidad de la actividad física, como cuestionarios, equipamientos o instrumentos observacionales, los cuales son válidos y confiables (McKenzie \& van der Mars, 2015). En este sentido, para México, en el ámbito escolar, las clases de educación física son el espacio ideal para fomentar la práctica de actividad física, dado que se imparten 50 minutos, dos veces por semana, con enfoque didáctico global de la motricidad, donde se pretende que el alumno asuma un rol protagónico, explore y viva experiencias motrices con sus compañeros, asumiendo códigos compartidos de conducta y comunicación, y conozca su corporeidad, habilidades y destrezas motrices, por lo que se concibe un alumno crítico, reflexivo, analítico y propositivo, tanto en la escuela como en los diferentes ámbitos de actuación en los que se desenvuelve (SEP, 2012).

A su vez, el programa de estudios 2011 de Educación Física asume, dentro de sus ejes pedagógicos, el denominado Valores, género e interculturalidad, del cual forma parte la equidad de género, que propicia establecer una convivencia más equitativa e igualitaria entre niñas y niños, donde se valore a la persona por sus capacidades y potencialidades (SEP, 2012). Por otra par- 
te, la Organización de las Naciones Unidas para la Educación, la Ciencia y la Cultura (UNESCO), señala que la escuela es un elemento clave para proporcionar a las niñas la información, competencias y confianza necesarias para la práctica de la actividad física y del deporte durante toda la vida (UNESCO, 2015).

De acuerdo con nuestro conocimiento, no existen referencias en México que valoren la intensidad de la actividad física mediante la frecuencia cardiaca con pulsómetros y la tabla de clasificación gráfica de esfuerzo para niños. Por lo anterior, el objetivo de la presente investigación fue comparar, de acuerdo con el género, el índice de actividad física moderada a vigorosa, la tasa de gasto energético, el promedio de frecuencia cardiaca y la percepción de esfuerzo en estudiantes de tercero y cuarto grado de primaria, durante la clase de educación física y el recreo.

\section{MÉTODO}

\section{Muestra y selección de participantes}

El estudio fue aprobado y registrado por la Coordinación de Posgrado e Investigación de la Universidad Autónoma de Baja California (Protocolo \#UABC-EXB-225), y se llevó a cabo entre enero y diciembre de 2016, bajo un diseño metodológico transversal comparativo, con muestreo no probabilístico por conveniencia (Thomas et al., 2001), solicitando anuencia de participación a los directivos, profesores, padres de familia y estudiantes de una escuela primaria pública de la ciudad de Mexicali, Baja California, explicando los detalles, beneficios y compromisos de la investigación mediante carta de consentimiento, programando horarios de evaluación y siguiendo los principios éticos de investigación en seres humanos de la declaración de Helsinki (Puri et al., 2009). En total, participaron sesenta y cinco estudiantes ( $\mathrm{n}=33$ hombres y $\mathrm{n}=32$ mujeres) pertenecientes a tercero $\mathrm{y}$ cuarto grado, con edad de $8.7 \pm 0.4$ años. 


\section{Procedimientos}

Las variables del estudio de índice de actividad física moderada a vigorosa, tasa de gasto energético, promedio de frecuencia cardiaca y percepción de esfuerzo, fueron evaluadas en la clase de educación física y recreo de la siguiente manera: las clases de educación física se planearon y aplicaron por 50 minutos cada una, en el horario establecido en el centro escolar, dos días a la semana (martes y jueves), aplicándose en total 16 clases (8 para cada grado), bajo el enfoque didáctico con la guía para el maestro educación básica en primaria de tercero y cuarto grado, de acuerdo al programa de estudio 2011 de la SEP. En tercer grado se aplicó el bloque I: Mi cuerpo en el espacio: combinando acciones. En cuarto grado aplicó el bloque I: No hacen falta alas, saltando ando. Dos profesores de educación física organizaron los contenidos por secuencias de trabajo, con estrategias que favorecían las competencias y aprendizajes esperados. El recreo se llevó a cabo de manera ordinaria, con los 30 minutos establecidos en el horario de la escuela, $y$ al igual que las variables evaluadas en la clase de educación física, se midieron los dos días a la semana (martes y jueves) durante cuatro semanas.

Para evaluar la intensidad durante la clase de educación física y el recreo, se utilizó el instrumento System for Observing Fitness and Instruction Time (SOFIT) (Sistema para observar el tiempo de instrucción de actividad física) (Mckenzie et al., 1992; Mckenzie, 2002). La metodología se realizó con dos observadores entrenados, quienes seleccionaron al azar, por sorteo sencillo, a 4 estudiantes (2 hombres y 2 mujeres), basados en la lista del grupo, usando el esquema de procedimientos en el manual SOFIT; en tiempos separados, la clase de educación física y el recreo fueron observados en secuencia rotatoria de 12 intervalos durante 20 segundos cada uno, repitiéndose las observaciones durante toda la clase y el recreo, siguiendo un audio que indicó los tiempos de registro de la actividad. Para llevar a cabo esta actividad, se utilizó como instrumento un reproductor MP3 Samsung YP-U6AB. 
Para determinar la intensidad, se usaron códigos para clasificar los niveles de actividad, los cuales permitieron estimar el gasto energético asociado con la actividad física. Este procedimiento se clasificó en cinco códigos: 1) acostado, 2) sentado, 3) parado, 4) caminando, y 5) muy activo, correspondiente a correr o a una actividad con un gasto energético mayor. A partir de la cuantificación de estos códigos, se estableció el índice de actividad física moderada a vigorosa (IAFMV), sumando porcentualmente los códigos 4) caminando y 5) muy activo, del total del tiempo de la clase de educación física y el recreo. Siguiendo los procedimientos del SOFIT, la tasa de gasto energético en $\mathrm{kcal} / \mathrm{kg} / \mathrm{min}$ se calculó a partir de los valores de tiempo en los códigos: 1) acostado, 2) sentado, 3) parado, 4) caminando, y 5) muy activo, mediante la fórmula TGE $(\mathrm{kcal} / \mathrm{kg} / \mathrm{min})$ = fracción acostado x $0.029 \mathrm{kcal} /$ $\mathrm{kg} / \mathrm{min}$ + fracción sentado $\times 0.047 \mathrm{kcal} / \mathrm{kg} / \mathrm{min}$ + fracción parado x $0.051 \mathrm{kcal} / \mathrm{kg} / \mathrm{min}$ + fracción caminando $\times 0.096 \mathrm{kcal} / \mathrm{kg} / \mathrm{min}$ + fracción muy activo x $0.144 \mathrm{kcal} / \mathrm{kg} / \mathrm{min}$. Los dos recolectores de datos fueron entrenados siguiendo el estándar del protocolo SOFIT, memorizando las definiciones operacionales de los códigos y aprendiendo los procedimientos metodológicos. Las medidas de confiabilidad fueron tomadas en el $100 \%$ de las observaciones (estadística Kappa 0.83).

Simultáneamente, a los 4 estudiantes seleccionados al azar (2 hombres y 2 mujeres) en la observación SOFIT, se les colocó un pulsómetro Polar bluetooth smart Inc, Finlandia ${ }^{\circledR}$, para monitorear la frecuencia cardiaca (lat/min) durante los 50 minutos de la clase de educación física, y posteriormente los 30 minutos del recreo, determinando así el promedio de la frecuencia cardiaca durante cada actividad.

La percepción del esfuerzo fue determinada mediante el Pictorial Children's Effort Rating Table (PCERT) (Apéndice D) (Tabla de clasificación gráfica de esfuerzo para niños), desarrollada por Yelling et al. (2002), con base en la escala CR-10 de Borg (Borg, 1982). Ajustándola al desarrollo cognitivo de los niños, 
añadieron figuras a la escala, haciendo más apropiada elegir una opción de un valor representativo para las edades infantiles con puntuaciones de 1 a 10, con un valor medio correspondiente a 5. La aplicación al mismo estudiante se realizó inmediatamente finalizada la educación física y el recreo.

\section{Análisis estadístico}

Con base en lo establecido en la introducción, y refiriendo datos de investigaciones realizadas en los contextos nacional e internacional en cuanto a las variables de estudio en estudiantes de escuelas primarias durante la clase de educación física y el recreo, la metodología utilizada en nuestra investigación estableció una prueba de hipótesis alterna y otra nula. El análisis estadístico se realizó mediante el Paquete Estadístico para las Ciencias Sociales (SPSS), versión 21.0 para Windows (IBM Corporation, New York, USA). Calculando los valores descriptivos de las variables, para verificar la normalidad de los grupos y la homogeneidad de la varianza de los datos, se utilizó el test estadístico de Shapiro-Wilk en las variables fijas, presentando un grado de significancia de P-Valor $\geq 0.05$. Como estudio transversal, al comparar como variables fijas el género de los estudiantes, y teniendo como variables aleatorias numéricas el índice de actividad física moderada a vigorosa, la tasa de gasto energético, la frecuencia cardiaca y la percepción de esfuerzo, se utilizó la prueba t Student para muestras independientes, con la finalidad de calcular la igualdad de la varianza, determinando un nivel de $\alpha \leq 0.05$, es decir un 5\% como porcentaje de error de la prueba estadística, procesando los datos en tablas y figuras.

\section{RESULTADOS}

En las tablas 1 y 2 se observa la estadística descriptiva media $(\mathrm{M})$, la desviación estándar $( \pm \mathrm{DE})$, la normalidad de los grupos 
y la homogeneidad de la varianza de las variables estudiadas durante la clase de educación física y el recreo.

Tabla 1. Estadística descriptiva y homogeneidad de la varianza de las variables de estudio, por género, en clase de educación física.

\begin{tabular}{|c|c|c|c|c|}
\hline \multirow{3}{*}{ Variables } & \multicolumn{4}{|c|}{ Clases de Educación Física } \\
\hline & \multicolumn{2}{|c|}{ Hombres } & \multicolumn{2}{|c|}{ Mujeres } \\
\hline & $M \pm D E$ & $\begin{array}{c}\text { Test } \\
\text { Shapiro-Wilk }\end{array}$ & $M \pm D E$ & $\begin{array}{c}\text { Test } \\
\text { Shapiro-Wilk }\end{array}$ \\
\hline 1) acostado (\%) & $2.5 \pm 0.6$ & 0.253 & $2.7 \pm 0.6$ & 0.129 \\
\hline 2) sentado (\%) & $12.1 \pm 5$ & 0.856 & $12.6 \pm 4.7$ & 0.410 \\
\hline 3) parado (\%) & $31.3 \pm 11.2$ & 0.442 & $38.2 \pm 12.5$ & 0.29 \\
\hline 4) caminando (\%) & $34.9 \pm 6.7$ & 0.110 & $32.3 \pm 8.3$ & 0.104 \\
\hline 5) muy activo (\%) & $19.2 \pm 7.3$ & 0.290 & $14.2 \pm 10.2$ & 0.118 \\
\hline $\begin{array}{l}\text { Índice de actividad física } \\
\text { moderada a vigorosa (\%) }\end{array}$ & $54.1 \pm 6.5$ & 0.416 & $46.5 \pm 9.1$ & 0.261 \\
\hline $\begin{array}{l}\text { Tasa de gasto } \\
\text { energético (kcal/kg/min) }\end{array}$ & $\begin{array}{c}0.0979 \pm \\
0.003\end{array}$ & 0.249 & $\begin{array}{c}0.0829 \pm \\
0.004\end{array}$ & 0.304 \\
\hline $\begin{array}{l}\text { Frecuencia cardiaca } \\
\text { promedio (lat/min) }\end{array}$ & $126.3 \pm 6.4$ & 0.721 & $119.6 \pm 6.2$ & 0.537 \\
\hline Percepción del esfuerzo & $5.2 \pm 1.60$ & 0.356 & $5.1 \pm 1.30$ & 0.581 \\
\hline
\end{tabular}

Tabla comparativa, por género, de la media, desviación estándar ( \pm ) normalidad y homogeneidad de la varianza entre grupos, mediante el test estadístico Shapiro-Wilk, * $p \geq 0.05$. Variables: Distribución porcentual de la intensidad de las clases de educación física, evaluada por SOFIT, clasificando los códigos como 1) acostado; 2) sentado; 3) parado; 4) caminando; y 5) muy activo, que corresponde a correr o una actividad con un gasto energético mayor. Índice de actividad física moderada a vigorosa (\%) suma porcentual de los códigos 4) caminando y 5) muy activo. Tasa de gasto energético en $\mathrm{kcal} / \mathrm{kg} / \mathrm{min}$, se calculó a partir de los valores de tiempo en todos los códigos. Promedio de frecuencia cardiaca en latidos por minuto FC (lat/min) (Polar Inc, Finlandia ${ }^{\circledR}$ ). Percepción del esfuerzo, determinada mediante el Pictorial Children's Effort Rating Table (PCERT). 
Tabla 2. Estadística descriptiva y homogeneidad de la varianza de las variables de estudio, por género, en recreo.

\begin{tabular}{|c|c|c|c|c|}
\hline \multirow{3}{*}{ Variables } & \multicolumn{4}{|c|}{ Recreo } \\
\hline & \multicolumn{2}{|c|}{ Hombres } & \multicolumn{2}{|c|}{ Mujeres } \\
\hline & $M \pm D E$ & $\begin{array}{c}\text { Test } \\
\text { Shapiro-Wilk }\end{array}$ & $M \pm D E$ & $\begin{array}{c}\text { Test } \\
\text { Shapiro-Wilk }\end{array}$ \\
\hline 1) acostado (\%) & $4.2 \pm 1.2$ & 0.531 & $3.1 \pm 0.9$ & 0.314 \\
\hline 2) sentado (\%) & $19.2 \pm 9.3$ & 0.267 & $21.6 \pm 8.8$ & 0.105 \\
\hline 3) parado (\%) & $30.2 \pm 12.2$ & 0.156 & $35 \pm 10.3$ & 0.129 \\
\hline 4) caminando (\%) & $32.2 \pm 10.3$ & 0.079 & $32.3 \pm 11.2$ & 0.301 \\
\hline 5) muy activo (\%) & $14.2 \pm 6.3$ & 0.454 & $8 \pm 3.6$ & 0.379 \\
\hline $\begin{array}{l}\text { Índice de actividad física } \\
\text { moderada a vigorosa (\%) }\end{array}$ & $46.4 \pm 10.6$ & 0.022 & $40.3 \pm 8.2$ & 0.771 \\
\hline $\begin{array}{l}\text { Tasa de gasto energético } \\
(\mathrm{kcal} / \mathrm{kg} / \mathrm{min})\end{array}$ & $\begin{array}{c}0.0613 \pm \\
0.002\end{array}$ & 0.295 & $\begin{array}{c}0.0554 \pm \\
0.003\end{array}$ & 0.529 \\
\hline $\begin{array}{l}\text { Frecuencia cardiaca } \\
\text { promedio (lat/min) }\end{array}$ & $117.6 \pm 12.6$ & 0.111 & $\begin{array}{c}114.3 \pm \\
13.2\end{array}$ & 0.77 \\
\hline Percepción del esfuerzo & $4.6 \pm 1.50$ & 0.901 & $4.5 \pm 1.80$ & 0.204 \\
\hline
\end{tabular}

Tabla comparativa, por género, de la media, desviación estándar ( \pm ) normalidad y homogeneidad de la varianza entre grupos mediante el test estadístico Shapiro-Wilk, $* p \geq 0.05$. Variables: Distribución porcentual de la intensidad en el recreo evaluada por SOFIT, clasificando los códigos como 1) acostado; 2) sentado; 3) parado; 4) caminando; y 5) muy activo. que corresponde a correr o una actividad con un gasto energético mayor. Índice de actividad física moderada a vigorosa (\%) suma porcentual de los códigos 4) caminando y 5) muy activo. Tasa de gasto energético en $\mathrm{kcal} / \mathrm{kg} / \mathrm{min}$, se calculó a partir de los valores de tiempo en todos los códigos. Promedio de frecuencia cardiaca en latidos por minuto FC (lat/min) (Polar Inc, Finlandia ®). Percepción del esfuerzo, determinada mediante el Pictorial Children's Effort Rating Table (PCERT).

En la figura 1 se muestra el resultado de la prueba t Student para muestras independientes, el cual mostró una P-Valor=.001 menor a $\alpha \leq 0.05$ en el índice de actividad física moderada a 
vigorosa durante la clase de educación física en los niños

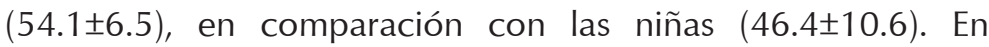
los valores durante el recreo por género no se encontraron diferencias significativas (0.177).

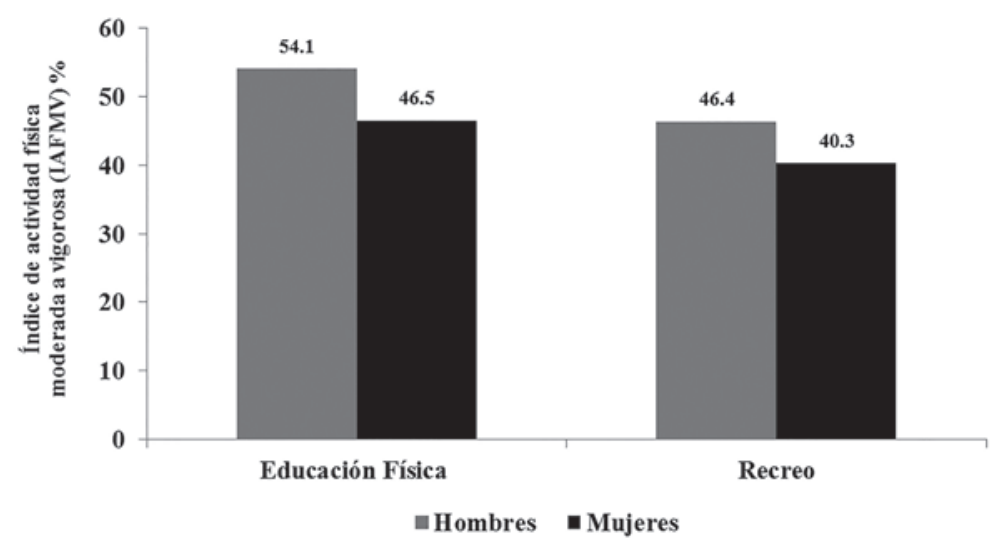

Figura 1. Índice de actividad física moderada a vigorosa (IAFMV) en $\%$, de los participantes, durante la clase de educación física y el recreo, de acuerdo al género.

Promedio porcentual del índice de actividad física moderada a vigorosa IAFMV = a la suma porcentual de los códigos 4) caminando y 5) muy activo, que corresponde a correr o una actividad con un gasto energético mayor, del total del tiempo de las clases de educación física y el recreo, evaluado mediante el sistema para observar el tiempo de instrucción de actividad física SOFIT (Mckenzie et al., 1992).

La figura 2 refleja los resultados comparativos por género de la tasa de gasto energético durante la clase de educación física, los cuales fueron significativamente mayores en hombres $(0.0979 \pm 0.003)$ que en mujeres $(0.0829 \pm 0.004)$, con una P-Valor=.002 menor a $\alpha \leq 0.05$. Durante el recreo, de acuerdo con el género, los valores de la prueba t Student para muestras independientes no encontraron diferencias significativas, presentando un nivel de significancia de 0.209 . 


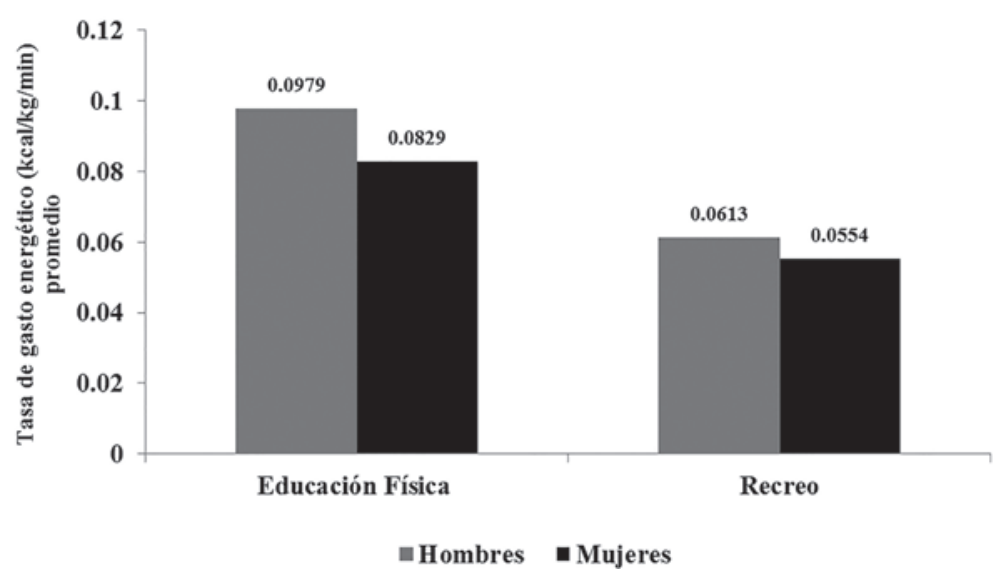

Figura 2. Tasa de gasto energético ( $\mathrm{kcal} / \mathrm{kg} / \mathrm{min}$ ) promedio de los participantes durante la clase de educación física y el recreo, de acuerdo al género.

La Tasa de Gasto Energético en $\mathrm{kcal} / \mathrm{kg} / \mathrm{min}$ se calculó a partir de los valores de tiempo en los códigos: 1) acostado, 2) sentado, 3) parado, 4) caminando, y 5) muy activo, mediante la fórmula TGE $(\mathrm{kcal} / \mathrm{kg} / \mathrm{min})$ = fracción acostado $x$ $0.029 \mathrm{kcal} / \mathrm{kg} / \mathrm{min}$ + fracción sentado $\times 0.047 \mathrm{kcal} / \mathrm{kg} / \mathrm{min}$ + fracción parado $\mathrm{x}$ $0.051 \mathrm{kcal} / \mathrm{kg} / \mathrm{min}$ + fracción caminando $\times 0.096 \mathrm{kcal} / \mathrm{kg} / \mathrm{min}$ + fracción muy activo x $0.144 \mathrm{kcal} / \mathrm{kg} / \mathrm{min}$, mediante el sistema para observar el tiempo de instrucción de actividad física SOFIT (Mckenzie et al., 1992).

Los valores calculados al monitorear la frecuencia cardiaca por género se muestran en la figura 3. La igualdad de la varianza mediante la prueba t Student para muestras independientes resultó con un nivel de significancia de P-Valor=.005 $\alpha \leq 0.05$ en la clase de educación física, con promedio significativamente mayor

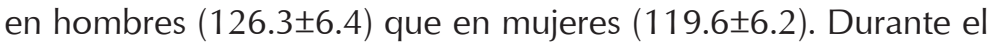
recreo, de acuerdo con el género, no se encontraron diferencias significativas, presentando un nivel de significancia de 0.516 .

En la figura 4 se muestran los promedios, por género, de la percepción del esfuerzo. Los resultados en la prueba t Student para muestras independientes fueron valores no significativos de 0.312 y 0.317 , en educación física y recreo respectivamente. 


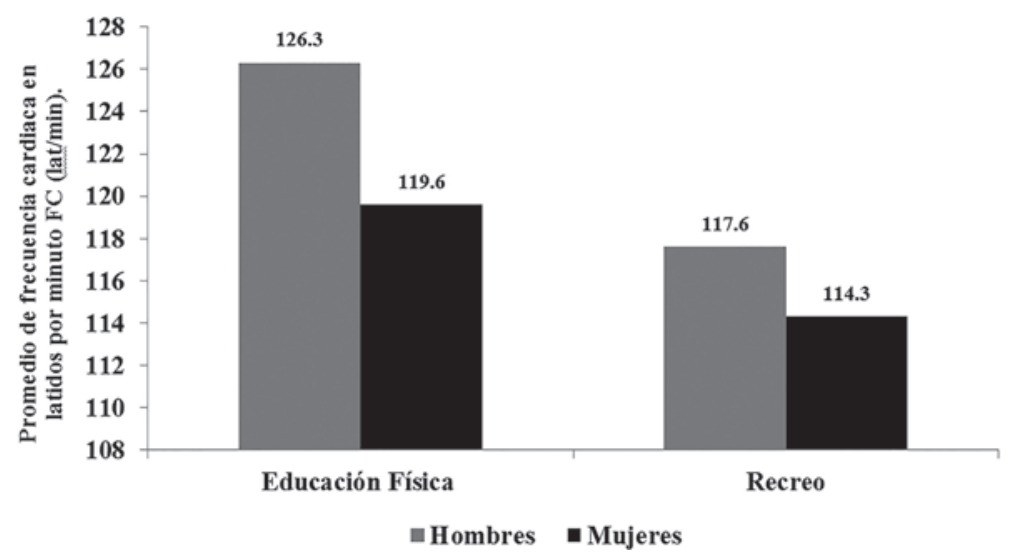

Figura 3. Frecuencia cardiaca (lat/min) promedio de los participantes, durante la clase de educación física y el recreo, de acuerdo al género.

Promedio de frecuencia cardiaca en latidos por minuto FC (lat/min), variables medidas con monitor de frecuencia cardiaca bluetooth smart (Polar Inc, Finlandia $\left.{ }^{\circledR}\right)$, monitoreada durante la clase de educación física y el recreo.

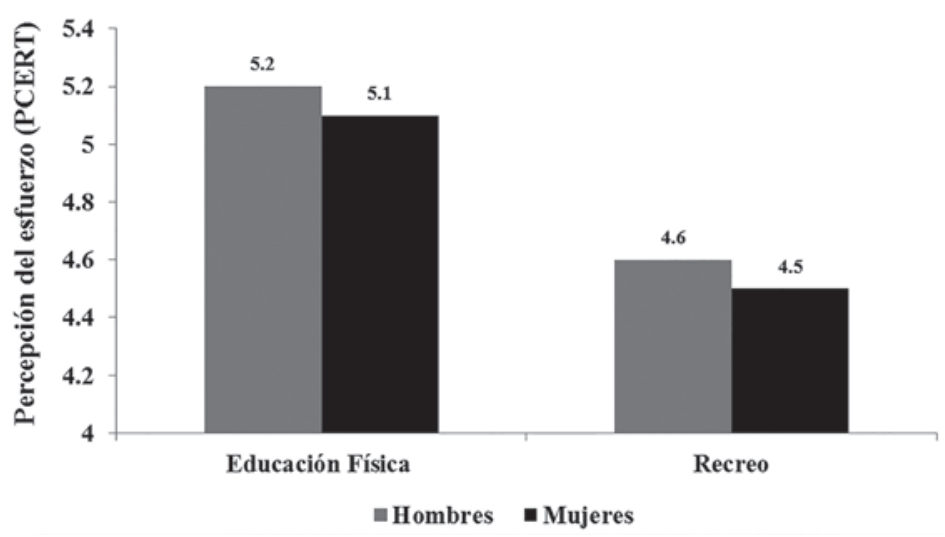

Figura 4. Percepción del esfuerzo promedio de los participantes durante la clase de educación y el recreo de acuerdo al género, evaluado por la tabla de clasificación gráfica de esfuerzo para niños (PCERT).

Percepción del esfuerzo, determinada mediante el Pictorial Children's Effort Rating Table (PCERT) (Tabla de clasificación gráfica de esfuerzo para niños), desarrollada por Yelling et al. (2002). 


\section{DISCUSIÓN Y CONCLUSIONES}

Al comparar las varianzas en las variables fijas por género durante la clase de educación física, con las variables aleatorias de índice de actividad física moderada a vigorosa (0.001), tasa de gasto energético (0.002) y promedio de frecuencia cardiaca (0.005), mostraron una P-Valor mayor a $\alpha \leq 0.05$, contrario a la percepción del esfuerzo (0.312). Con relación al recreo, en todas las variables no muestra diferencias significativas. Con base en lo anterior, de los sujetos estudiados, los hombres presentan, durante la clase de educación física, mayor intensidad de actividad física y gasto energético que las mujeres, aunque perciben el mismo esfuerzo. Los valores durante el recreo respecto a la intensidad de la actividad física, el gasto energético y la percepción del esfuerzo, resultaron iguales de acuerdo con el género.

En México se han realizado investigaciones en escuelas primarias, evaluando la intensidad de la actividad física en educación física, utilizando como instrumento el sistema para observar el tiempo de instrucción de actividad física SOFIT (Mckenzie, 2002; Mckenzie et al., 1992). Al revisar el estado del arte, se han reportado índices de actividad física moderada a vigorosa en educación física de $38.2 \%$, en una muestra de estudiantes de tercero y cuarto grado de primaria en Chihuahua, siendo mayor en niños (42.2\%) que en niñas (36.8\%) (Pérez, 2009). Otra investigación realizada en 12 escuelas de la Ciudad de México, con estudiantes de cuarto y quinto grado de primaria, reportó un $29.2 \%$ de intensidad moderada a vigorosa en clase de educación física (32.1\% niños y $25.7 \%$ niñas); de igual forma, dentro de los resultados se encontró, al evaluar mediante SOFIT a los estudiantes en el recreo, una intensidad de actividad física moderada y vigorosa de $40 \%$ (43\% niños y 36\% niñas) (Jennings et al., 2009). Investigaciones mencionan que, durante el tiempo del recreo, los estudiantes pueden alcanzar hasta el $40 \%$ de la actividad física diaria recomendada (Ridgers et al., 
2011). Se ha identificado en el recreo mayor participación en actividad física moderada a vigorosa de los niños con respecto a las niñas (Springer et al., 2013). Por lo anterior, el recreo se ha referido como una oportunidad de crear ambientes organizados para fomentar actividades que favorezcan el movimiento con equidad de género (Medina et al., 2015; Robinson et al., 2014). De acuerdo a la Secretaría de Educación Pública (SEP), durante el ciclo escolar se cuenta con 30 minutos de tiempo de recreo por jornada escolar, lo que se establece dentro de los lineamientos para la organización y el funcionamiento en escuelas en la educación básica, tiempo que los alumnos pueden destinar al juego libre y a la ingesta de un refrigerio durante la jornada escolar (SEP, 2014).

En el sistema educativo Mexicano, en comparación con otros contextos internacionales, la intensidad de la actividad física no es un factor determinante para valorar el proceso educativo y la salud de los niños en la escuela (Banville, 2006; NASPE, 2009; WHO, 2014), aunque el programa de estudios 2011 de Educación Física asume, dentro de sus ejes pedagógicos, la equidad de género (SEP, 2012); de igual manera, con la intención de garantizar un enfoque inclusivo la educación física, la UNESCO recomienda atender todas las medidas apropiadas para eliminar la discriminación contra la mujer, y asegurar las mismas oportunidades para participar activamente en el deporte y la educación física (UNESCO, 2015). En ese sentido, los resultados del presene estudio no reflejan una actividad física igualitaria entre hombres y mujeres durante la clase educación física.

Por otra parte, se entiende que existen múltiples factores ambientales que pueden determinar la intensidad de la actividad física, el gasto energético y la percepción del esfuerzo de los estudiantes en la clase de educación física y el recreo (Hernández et al., 2010; Hollis et al., 2016; Skala et al., 2012), pero, al analizar resultados de estudios realizados en México con similares procedimientos metodológicos, utilizando como instrumento de 
evaluación el SOFIT, corroboran los resultados encontrados en esta investigación. Por ejemplo, la evaluación a estudiantes de tercero y cuarto grado de primaria en Chihuahua, refiere mayor actividad física moderada a vigorosa en niños (42.2\%) que en niñas (36.8\%) (Pérez, 2009). Otra investigación realizada en la Ciudad de México en estudiantes de cuarto y quinto grado de primaria, reportó una intensidad moderada a vigorosa durante la clase de educación física de $32.1 \%$ en niños, y $25.7 \%$ en niñas (Jennings et al., 2009). Otra investigación en Ciudad de México, con estudiantes de tercero y quinto grado de primaria, mostró un índice de actividad física moderada a vigorosa de $44 \%$ en niños y $38 \%$ en niñas (Gharib et al., 2015). De este modo, se confirma que, a pesar de que las clases de educación física son diseñadas y planeadas con base en la guía de un programa educativo, existen factores que propician un mayor movimiento por parte de los estudiantes de género masculino. Por otra parte se destaca, al analizar los porcentajes de actividad física moderada a vigorosa durante la clase de educación física, que no se alcanzaron estándares establecidos internacionalmente por la NASPE, con la participación de los estudiantes en una intensidad moderada a vigorosa por lo menos del $50 \%$ del tiempo de la clase de educación física (Banville, 2006). Los resultados en nuestro estudio, parcialmente se contradicen a los anteriores, ya que muestran mayores valores de índice de actividad física moderada a vigorosa en los niños, con 54\%, lo que puede obedecer a que, en los estudios citados, la metodología fue evaluar de manera transversal en una ocasión la clase de educación física, mientras que, en el presente estudio, se evaluó en ocho ocasiones a los mismos dos grupos, pudiendo ello tener influencia. Sin embargo, en nuestros resultados se sigue reflejando una participación de las estudiantes de género femenino de $46 \%$, inferior a la recomendada por la NASPE (Banville, 2006; NASPE, 2009). Con relación al recreo, nuestros valores fueron mayores en niños $(46 \%)$ que en niñas $(40.3 \%)$, pero no significativos. Estudios 
reportan porcentajes similares durante el recreo en México, con una intensidad moderada a vigorosa de $40 \%$ (43\% niños, $36 \%$ niñas) (Jennings et al., 2009). Otro estudio que evaluó a 83 mujeres estudiantes de secundaria (edad 13 a 15 años) en el recreo, refirió un 33.7\% (Medina et al., 2015).

La relevancia del gasto energético por actividad física como factor asociado a la salud, recomendadas por la OMS y las guías alimentarias y de actividad física en contexto de sobrepeso y obesidad en la población mexicana, no diferencia el género, es decir, recomienda en niños y niñas acumular por igual 60 minutos diarios de actividad física moderada a vigorosa (Bonvecchio et al., 2015; WHO, 2014). En el presente estudio existieron, en mayor medida, diferencias significativas de gasto energético de los niños respecto a las niñas durante la clase de educación física y el recreo. En México existen referencias que evaluaron en educación física el gasto energético mediante podómetros, reportando un promedio de gasto calórico en educación física de 164.21 Kcal en estudiantes de quinto y sexto grado de la ciudad de Colima (Flores et al., 2016). Otra investigación en el contexto educativo mexicano, realizada en Ciudad Juárez, evaluó el gasto calórico en niños de cuarto a sexto grado de primaria mediante podómetros en el recreo, refiriendo, en cuanto a género, $87.35 \mathrm{Kcal} / 30 \mathrm{~min}$ en niños, y $83.95 \mathrm{Kcal} / 30 \mathrm{~min}$ en niñas (Zúñiga et al., 2016). Investigaciones han identificado que los niños, durante el recreo, realizan mayor actividad física moderada a vigorosa que las niñas (Springer et al., 2013); al comparar estos resultados con investigaciones realizadas en México, a pesar de haber utilizado metodología de evaluación diferente, concuerdan con los de nuestra investigación (Zúñiga et al., 2016; Flores et al., 2016).

La frecuencia cardiaca fue significativamente mayor en los niños que en las niñas. Para conocer los rangos funcionales de trabajo físico, se utilizó la fórmula establecida por el Colegio 
Americano de Medicina del Deporte (ACSM) para establecer la frecuencia cardiaca máxima (FCmáx) en niños, calculándose de la siguiente manera: FCmáx=206.9-(edad en años×0.67) (ACSM, 2011). De esta forma se determinaron los umbrales de esfuerzo físico, resultando en clase de educación física en los niños de $62 \%$ y en las niñas de $58 \%$; de acuerdo con las guías de prescripción del ejercicio del ACSM, el porcentaje promedio de los hombres representa una intensidad de actividad física clasificada como aceptable, y el de las mujeres se clasifica en un umbral bajo, para poder obtener beneficios de salud como resultado de la actividad física (ACSM, 2011). Durante el recreo, los valores de frecuencia cardiaca promedio resultaron no significativos, y los porcentajes de esfuerzo físico fueron de $57 \%$ en niños y $56 \%$ en niñas. En la percepción del esfuerzo, evaluada mediante la tabla de clasificación gráfica de esfuerzo, nuestros datos no muestran consistencia con los reportados por Hernández et al. (2010), quienes refieren diferencias significativas por género, lo que se puede explicar porque la intensidad en la clase de educación física en esta investigación fue menor a la realizada en ese estudio. Por otra parte, en nuestra investigación, los valores de la frecuencia cardiaca no fueron equivalentes a los establecidos en la tabla de clasificación gráfica de esfuerzo, donde valores de percepción por arriba de 5 representan frecuencias cardiacas entre 130 y 140 (lat/min), dado que fueron evaluadas con una ecuación diferente, por lo cual podría existir un sesgo para la edad con relación a la metodología utilizada en nuestro estudio, con ecuaciones para niños (ACSM, 2011; Yelling et al., 2002).

Como conclusión, al identificar menor intensidad de actividad física en mujeres durante la clase de educación física, se recomienda, con base en la guía del programa de estudios SEP 2011, que los profesionales de la educación física realicen una intervención docente con estrategias didácticas y contenidos que, equitativamente, involucren en el movimiento a los 
estudiantes de ambos géneros, propiciando así una educación física de calidad, como lo establece la UNESCO.

\section{REFERENCIAS}

1. ACSM American College of Sports Medicine (2011). Complete guide to fitness \& health. USA: ACSM.

2. Banville, D. (2006). Analysis of exchanges between novice and cooperating teachers during internships using the NCATE/NASPE standards for teacher preparation in physical education as guidelines. Research Quarterly for Exercise and Sport, 77(2), 208-221.

3. Bonvecchio, A., Fernández, A., Plazas, M., Kaufer, M., Pérez, A., \& Rivera, J. (2015). Guías alimentarias y de actividad física en contexto de sobrepeso y obesidad en la población mexicana. Ciudad de México: Academia Nacional de Medicina.

4. Borg, G. (1982). Psychophysical bases of perceived exertion. Medicine \& Science in Sports \& Exercise, 14(5), 377-381.

5. Brooke, H., Corder, K., Atkin, A., \& van Sluijs, E. (2014). A systematic literature review with meta-analyses of within-and between-day differences in objectively measured physical activity in school-aged children. Sports Medicine, 44(10), 27-38.

6. Flores, P., Salazar, C., Gómez, J., Barreto, Y., Valdovinos, O., Vicente, J., \& Del Río, J. (2017). Medición del tiempo efectivo de la clase de educación física y su impacto en el gasto calórico en escolares de nivel primaria del municipio de Colima, México. Sportis. Revista Técnico-Científica del Deporte Escolar, Educación Física y Psicomotricidad, 3, 34-49.

7. Gharib, H., Galaviz, K., Lee, R., Safdie, M., Tolentino, L., \& Barquera, S. (2015). The influence of physical education lesson context and teacher behaviour on student physical activity in Mexico. RETOS Nuevas Tendencias en Educación Física, Deporte y Recreación, 28, 160-164.

8. Hernández, J., del Campo, J., Martínez, V., \& Moya, J. (2010). Percepción de esfuerzo en Educación Física y su relación con las directrices sobre actividad física. Revista Internacional de Medicina y Ciencias de la Actividad Física y el Deporte, 10(40), 609-619.

9. Hollis, J., Williams, A., Sutherland, R., Campbell, E., Nathan, N., Wolfenden, L., \& Wiggers, J. (2016). A systematic review and meta-analysis of moderate-to-vigorous physical activity levels in elementary school physical education lessons. Preventive Medicine, 86, 34-54.

10. Honas, J., Washburn, R., Smith, B., Greene, J., Cook, G., \& Donnelly, J. (2008). The System for Observing Fitness Instruction Time (SOFIT) 
as a measure of energy expenditure during classroom-based physical activity. Pediatric Exercise Science, 20(4), 439-445.

11. INSP Instituto Nacional de Salud Publica (2016). Encuesta Nacional de Salud y Nutrición Medio Camino 2016 ENSANUT MC 2016. México: INSP. Disponible en: http://ensanut.insp.mx/

12. Jennings, N., Nava, F., Bonvecchio, A., Safdie, M., González, I., Gust, T., \& Rivera, J. (2009). Physical activity during the school day in public primary schools in Mexico City. Salud Pública de México, 51(2), 141-147.

13. Kim, J. (2012). Are physical education-related state policies and schools' physical education requirement related to children's physical activity and obesity? Journal of School Health, 82(6), 268-276.

14. Langford, R., Bonell, C., Jones, H., Pouliou, T., Murphy, S., Waters, E., Komro, K., Gibbs, L., Magnus, D., \& Campbell, R. (2015). The World Health Organization's Health Promoting Schools framework: a Cochrane systematic review and meta-analysis. BMC Public Health, 15:130.

15. Lonsdale, C., Rosenkranz. R., Peralta, L., Bennie, A., Fahey, P., \& Lubans, D. (2013). A systematic review and meta-analysis of interventions designed to increase moderate-to-vigorous physical activity in school physical education lessons. Preventive Medicine, 56(2), 152-161.

16. McKenzie, T. (2002). SOFIT. System for Observing Fitness Instruction Time. Overview and training manual. San Diego, CA: San Diego State University.

17. Mckenzie, T., Sallis, J., \& Nader, P. (1992). Sofit-System for Observing Fitness Instruction Time. Journal of Teaching in Physical Education, 11(2), 195-205.

18. McKenzie, T., Baquero, B., Crespo, N., Arredondo, E., Campbell, N., \& Elder, J. (2008). Environmental correlates of physical activity in Mexican-American children at home. Journal of Physical Activity and Health, 5(4), 579-591.

19. McKenzie, T., \& van der Mars, H. (2015). Top 10 research questions related to assessing physical activity and its contexts using systematic observation. Research Quarterly for Exercise and Sport, 86(1), 13-29.

20. Medina, C., Barquera, S., Katzmarzyk, P., \& Janssen, I. (2015). Physical activity during recess among 13-14 year old Mexican girls. BMC Pediatrics, 15(17), 2-8.

21. NASPE National Association for Sport and Physical Education (2009). National standards. Guidelines and position statements. USA: NASPE. Available from: http://www.aahperd.org/naspe/

22. Nettlefold, L., McKay, H., Warburton, D., McGuire, K., Bredin, S., \& Naylor, P. (2011). The challenge of low physical activity during the school day: at recess, lunch and in physical education. British Journal of Sports Medicine, 45(10), 813-819. 
23. Pérez, A. (2009). Impacto de la clase de educación física sobre la actividad moderada y vigorosa en niños de primaria. Revista Mexicana de Investigación en Cultura Física y Deporte, 1(1), 150-172.

24. Puri, K., Suresh, K., Gogtay, N., \& Thatte, U. (2009). Declaration of Helsinki implications for stakeholders in research. Journal of Postgraduate Medicine, 55(2), 131-134.

25. Ridgers, N., Carter, L., Stratton, G., \& McKenzie, T. (2011). Examining children's physical activity and play behaviors during school playtime over time. Health Education Research, 26(4), 586-595.

26. Robinson, L., Wadsworth, D., Webster, E., \& Bassett, D. (2014). School reform: The role of physical education policy in physical activity of elementary school children in Alabama's Black Belt Region. American Journal of Health Promotion, 28(3), 72-76.

27. SEP Secretaría de Educación Pública (2011). Programas de estudio 2011. Guía para el Maestro. Educación Básica. Primaria. México: Dirección General de Desarrollo Curricular (DGDC) y Dirección General de Formación Continua de Maestros en Servicio. Disponible en: http://www.centrodemaestros.mx/programas-de-estudio/

28. SEP Secretaría de Educación Pública (2014). Lineamientos para la organización y el funcionamiento de las escuelas de tiempo completo. México: SEP. Disponible en: http://basica.sep.gob.mx/seb2010/pdf/ MCTE/3LiORFunETCEduPri.pdf

29. Shilton, T. (2008). Creating and making the case: global advocacy for physical activity. Journal of Physical Activity and Health, 5(6), 765-776.

30. Sims, J., Scarborough, P., \& Foster, C. (2015). The effectiveness of interventions on sustained childhood physical activity: A systematic review and meta-analysis of controlled studies. PLoS One, 10(7), e0132935.

31. Skala, K., Springer, A., Sharma, S., Hoelscher, D., \& Kelder, S. (2012). Environmental characteristics and student physical activity in PE class: findings from two large urban areas of Texas. Journal of Physical Activity and Health, 9(4), 481-491.

32 Smith, N., Lounsbery, M., \& McKenzie, T. (2014) Physical activity in high school physical education: impact of lesson context and class gender composition. Journal of Physical Activity and Health, 11(1), 127-135.

33. Springer, A., Tanguturi, Y., Ranjit, N., Skala, K., \& Kelder, S. (2013). Physical activity during recess in low-income third-grade Texas students. American Journal of Health Behavior, 37(3), 318-324.

34. Story, M., Nanney, M., \& Schwartz, M. (2009). Schools and obesity prevention: creating school environments and policies to promote healthy eating and physical activity. Milbank Quarterly, 87(1), 71-100.

35. Thomas, J., Nelson, J., \& Silverman, S. (2001). Research methods in physical activity ( $6^{\text {th }} \mathrm{Ed}$.). Champaign, llinois: Human Kinetics. 
36. UNESCO Organización de las Naciones Unidas para la Educación, la Ciencia y la Cultura (2015). Educación física de calidad guía para los responsables políticos. París, Francia: UNESCO. Disponible en: http:// unesdoc.unesco.org/images/0023/002313/231340S.pdf

37. WHO World Health Organization (2014). Global strategy on diet, physical activity and health, physical activity and young people. Recommended levels of physical activity for children aged 5-17 years. Geneva, Switzerland: WHO. Available from: http://www.who.int/ dietphysicalactivity/factsheet_young_people/en/

38. Yates, T., Wilmot, E., Khunti, K., Biddle, S., Gorely, T., \& Davies, M. (2011). Stand up for your health: Is it time to rethink the physical activity paradigm? Diabetes Research and Clinical Practice, 93(2), 292-294.

39. Yelling, M., Lamb, K., \& Swaine, I. (2002). Validity of a pictorial perceived exertion scale for effort estimation and effort production during stepping exercise in adolescent children. European Physical Education Review, 8, 157-175.

40. Zúñiga, U., Ramos, A., Cervantes, M., \& Hernández, R. (2016). Determinación de la intensidad y el gasto calórico de la actividad física durante el recreo escolar en niños y niñas de primaria. E-motion Revista de Educación, Motricidad e Investigación, 6, 19-27. 